\title{
On the Origin of the Satellite Peaks in Alpha Particle Spectra
}

\author{
F. Camargo ${ }^{1}$, Carmen C. Bueno ${ }^{1,2}$, Josemary A. C. Gonçalves ${ }^{1,2}$, P. F. P. Rato Mendes ${ }^{3}$, \\ J. K. C. Pinto ${ }^{4}$, J. P. Souza ${ }^{4}$, and M. D. S. Santos ${ }^{1}$ \\ ${ }^{1}$ Instituto de Pesquisas Energéticas e Nucleares - IPEN-CNEN/SP, \\ CP. 11409, CEP 05422-970, São Paulo, SP, Brazil \\ ${ }^{2}$ Departamento de Física, Pontifícia Universidade Católica de São Paulo, PUC/SP, \\ R. Marquês de Paranaguá 111, CEP: 01303-050, São Paulo, SP, Brazil \\ ${ }^{3}$ LIP, Av. E. Garcia, P-1000 Lisbon, Portugal \\ ${ }^{4}$ Laboratório de Micro Eletrônica, EPUSP, CP. 61548, CEP 05424-970, São Paulo, SP, Brazil
}

Received on 13 October, 2003

\begin{abstract}
In this work we describe the results obtained with S3590-06 and ion-implanted diodes for alpha particle spectrometry. Satellite peaks were also investigated in order to identify not only the origin of these phenomena, but also the importance of the guard ring region on their relative intensities. The preliminary results indicate that the true cause of the spurious satellite peaks might be associated with the weak electric fields around the guard rings region and changes on the entrance window absorption near the edge of the diodes. Further steps are needed to clear up this problem.
\end{abstract}

\section{Introduction}

The widespread study on silicon diodes performance in the spectrometry of charged particles is due to the possibility of their use as a research tool, both in laboratory and in industrial applications [1-19], as the measurement of the isotopic ratios between chemical elements. In order to employ this technique in studies related to reactor fuel elements and their properties after irradiation, we have been studying the response of silicon diodes for alpha spectrometry. In spite of having very good energy resolution when used for heavy charged particle spectrometry, the silicon diodes usually give rise to some spurious peaks, developed at somewhat lower energies than the main peaks in the spectra. These "satellite" or "ghost" peaks were initially thought to be due to incomplete charge collection in weak electric field region [20-21] around the edges of the diode. So, one should expect that the relative intensity of these satellite peaks would increase with the number of the guard rings of the diode. Having in mind this perspective, we carried out some alpha spectra using two diodes: a commercial PIN photodiode (Hamamatsu-S3590) and an implanted silicon diode (type $\mathrm{Al} / \mathrm{p}^{+} / \mathrm{n} / \mathrm{n}^{+} / \mathrm{Al}$ ) which enable us to select from one to ten guard rings. This diode, developed in the framework of $\mathrm{R} \& \mathrm{D}$ programs for rad-hard silicon detectors to be used in the future CMS experiments at CERN, will be referred to below as CERN diode. Our preliminary results, with both diodes, show that the energy resolution for alpha particles from ${ }^{239} \mathrm{Pu},{ }^{241} \mathrm{Am}$ and ${ }^{244} \mathrm{Cm}$ in a mixed alpha source were reasonably good and the intensity of satellite peaks were about $4 \%$ compared to the true peaks, even when ten guard rings were connected to the ground.

\section{Experimental Set-Up}

The main characteristics of S3590-06 and CERN diodes, which are presented in Table I, were obtained from dynamic measurements of its capacitance and leakage current as a function of the reverse applied voltage. Since the CERN diode was supplied without electrodes (Fig. 1), its electric leads, as well as the guard rings connections, were made at Microelectronic Laboratory in São Paulo University (LME/USP).

In order to use these diodes as alpha particle detectors, they were housed inside a stainless steel vacuum chamber and its signal electric leads were connected to the field effect transistor (FET) in the first stage of a charge sensitive preamplifier, based on an integrated circuit A250 from Amptek. The distance between the first stage of the charge sensitive preamplifier and the diode was kept as short as possible to minimize the noise contribution originated from parasitic capacitances. The pulses from the preamplifier were shaped and amplified by a linear amplifier (Ortec 572) with adjustable time constants and fed to a multichannel analyzer (Ortec Spectrum Ace).

\section{Experimental Results}

First, the detection efficiencies of S3590-06 and CERN diodes for heavy charged particles, as a function of the reverse applied voltage, were measured using an alpha source of ${ }^{241} \mathrm{Am}$. The results obtained (Figs. 2 and 3) show that the counting rate is practically independent of the polarization voltage. Therefore, one can conclude that the depletion layer of both diodes are thick enough to absorb almost all the energy of the incoming alpha particles. 
TABLE I. The main characteristics of S3590-06 and CERN diodes.

\begin{tabular}{ccccccc}
\hline \hline & $\begin{array}{c}\text { Diode Area } \\
\left(\mathrm{mm}^{2}\right)\end{array}$ & $\begin{array}{c}\text { Bias } \\
(\mathrm{V})\end{array}$ & $\begin{array}{c}\text { Capacitance } \\
(\mathrm{pF})\end{array}$ & $\begin{array}{c}\text { Density of Leakage Current } \\
\left(\mathrm{nA} / \mathrm{mm}^{2}\right)\end{array}$ & $\begin{array}{c}\text { Depletion Region } \\
(\mu \mathrm{m})\end{array}$ & $\begin{array}{c}\text { Relative Efficiency } \\
(\%)\end{array}$ \\
\hline S3590-06 & 81.0 & -30 & 25.1 & 0.6 & 342.3 & 98 \\
CERN & 2.5 & -40 & 1.8 & 6.6 & 151.7 & 100 \\
\hline \hline
\end{tabular}

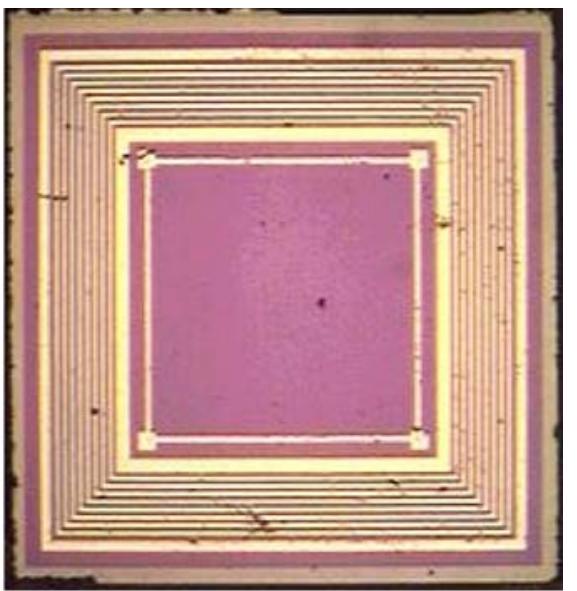

Figure 1 . The CERN diode photography.

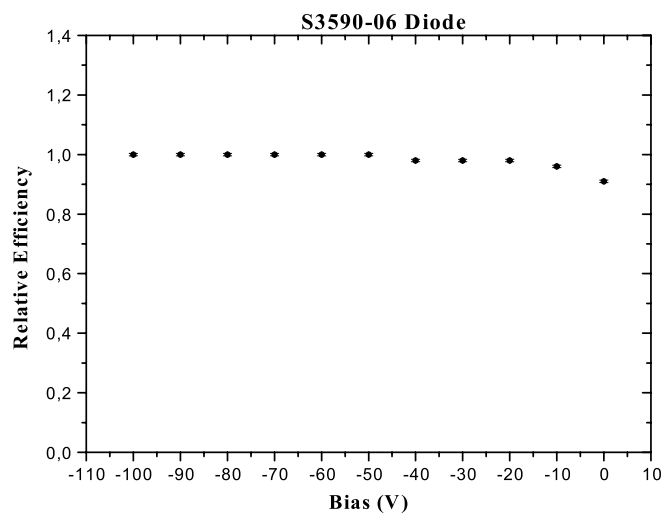

Figure 2. Relative efficiency of S3590-06 diode for alpha particle from ${ }^{241} \mathrm{Am}$.

In order to evaluate the influence of the bias voltage and shaping time constants on the alpha energy resolution, several spectra from a $5.5 \mathrm{kBq}$ mixed alpha source of ${ }^{239} \mathrm{Pu}$, ${ }^{241} \mathrm{Am}$ and ${ }^{244} \mathrm{Cm}$, placed at approximately $1.0 \mathrm{~cm}$ from the diodes, were recorded. The experimental results for both diodes showed that, even at room temperature and without reverse bias, it was possible to identify the main alpha group from each isotope. On the other hand, the effect of the voltage on the energy resolution was clearly observed: the fine structure lines of each isotope can be inferred from these measurements. In Figs. 4 and 5 the energy resolution for alpha particles from ${ }^{239} \mathrm{Pu},{ }^{241} \mathrm{Am}$ and ${ }^{244} \mathrm{Cm}$ are represented as a function of the time constants for both diodes.

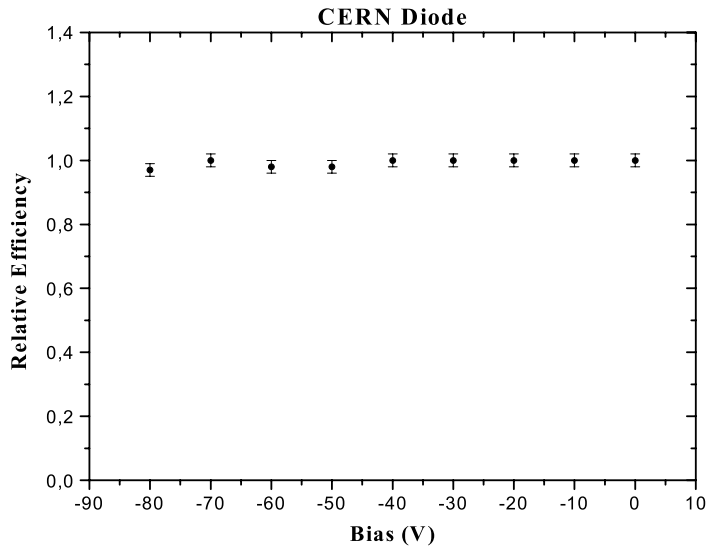

Figure 3. Relative efficiency of CERN diode for alpha particle from ${ }^{241} \mathrm{Am}$.

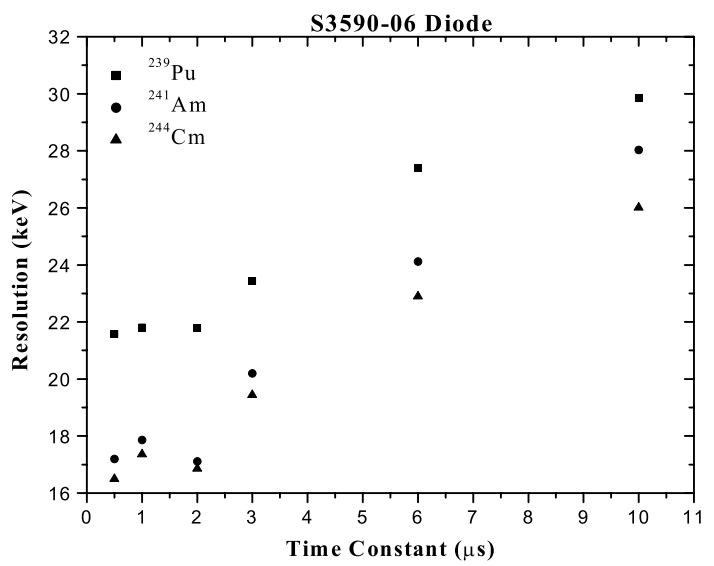

Figure 4. Energy resolution of S3590-06 diode as a function of time constant.

The best energy resolutions, of $17.11 \mathrm{keV}$ (S3590-06) and $23.39 \mathrm{keV}$ (CERN diode), for 5.486 MeV alpha particles from ${ }^{241}$ Am are shown in Figures 6 and 7, respectively. In spite of these very good energy resolutions, both diodes gave rise to several satellite peaks whose relative intensities (to the main groups) are affected by the bias voltage and time constants values. An analysis of the parameters associated to these spectra shows that, even though the CERN diode bears ten guard rings, the relative intensities of satellite peaks are lower than those obtained with S3590-06. 


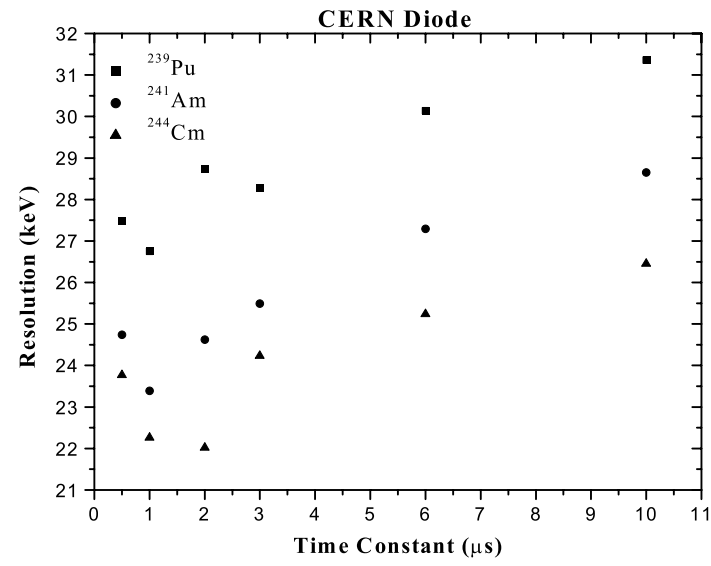

Figure 5. Energy resolution of CERN diode as a function of time constant.

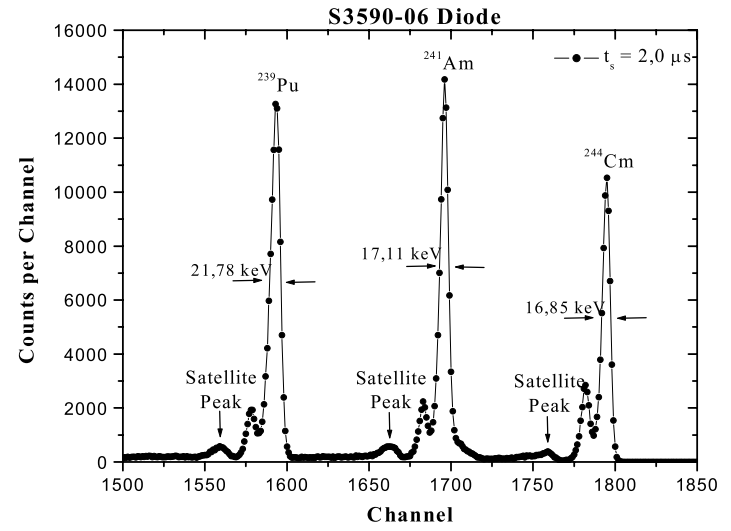

Figure 6. Alpha mixed source spectrum recorded with S3590-06 diode at room temperature and $-80 \mathrm{~V}$.

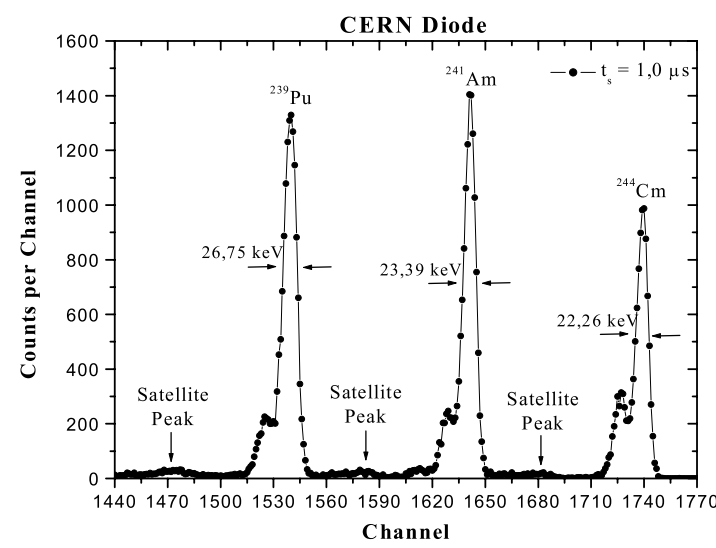

Figure 7. Alpha mixed source spectrum recorded with CERN diode at room temperature and $-60 \mathrm{~V}$.

\section{Conclusion}

Although both diodes studied gave rise to satellite peaks, they are particularly suitable for alpha particle spectroscopy and energy resolution comparable with surface barrier detectors can be achieved with these devices directly coupled to inexpensive preamplifiers. However, it is noteworthy to mention that the preliminary results indicate the true cause of the spurious satellite peaks might be not only associated with the weak electric field region around the guard rings, but also with changes on the entrance window absorption near the edge of the diodes. Further steps are needed to clear up this problem.

\section{Acknowledgments}

The authors would like to thank IPEN-CNEN/SP, CNPq and FAPESP for financial support during this work. One of us, F. Camargo, thanks CNPq for the award of a scholarship.

\section{References}

[1] L. Bellucci, Nucl. Instrum. and Meth. A462, 243 (2001).

[2] M. Pauluzzi, Nucl. Instrum. and Meth. A473, 67 (2001).

[3] F. Tosello, Nucl. Instrum. and Meth. A473, 210 (2001).

[4] Y.S. Tsyganov et al, Nucl. Instrum. and Meth. A477, 406 (2002).

[5] P. Sievers, Nucl. Instrum. and Meth. A485, 23 (2002).

[6] P.D. Bradley et al, Nucl. Instrum. and Meth. B 184, 135 (2001).

[7] M. Bruzzi et al, Nucl. Instrum. and Meth. A485, 172 (2002).

[8] E. do Couto and Silva, Nucl. Instrum. and Meth. A473, 107 (2001).

[9] G. Lutz et al, Nucl. Instrum. and Meth. A461, 393 (2001).

[10] A. Chambaudet et al, Radiation Measurements, 28, 127 (1997).

[11] M. Voytchev et al, Radiation Measurements, 31, 375 (1999).

[12] F.V. Tomé et al, Applied Radiation and Isotopes, 56, 393 (2002).

[13] G. Kraft, Nucl. Instrum. and Meth. A454, 1 (2000).

[14] N. A. Pavel, Nucl. Instrum. and Meth. A478, 1 (2002).

[15] G. Lindström et al, Nucl. Instrum. and Meth. A426, 1 (1999).

[16] T. Kamae, Nucl. Instrum. and Meth. A436, 297 (1999).

[17] A. Khodin et al, Nucl. Instrum. and Meth. A465, 253 (2001).

[18] T. Wichert and M. Deicher, Nucl. Instrum. and Meth. B693, 327 (2001).

[19] C.C. Bueno, J.A.C. Gonçalves and M.D.S. Santos, Nucl. Instrum. and Meth, A371, 460 (1996).

[20] D. Kollewe, Nucl. Instrum. and Meth. A254, 637 (1987).

[21] P.H. Gooda and W.B. Gilboy, Nucl. Instrum. and Meth. A255, 222 (1987). 